\title{
Saúde mental na grade curricular do curso de medicina em uma instituição de ensino superior: implantação das Diretrizes Curriculares Nacionais de 2014
}

Mental health in the curriculum of the medical course at a higher education institution: implementation of 2014 National Curricular Guidelines

Salud mental en el plan de estudios de la carrera de medicina en una institución de educación superior: implementación de las Directrices Curriculares Nacionales de 2014

Fernanda Penido Gonçalves de Souza ${ }^{1 *}$, Murilo de Miranda Cardoso1, Marcela Luíza Alves Pereira $^{1}$, Gabriel de Almeida Giardini ${ }^{1}$, Cristiane Reis dos Santos ${ }^{1}$, Larissa Daher Michel ${ }^{1}$, Laís Campos Gontijo ${ }^{1}$, Marina Brettas Tavares ${ }^{1}$, Estefânia Pereira Diniz ${ }^{1}$, Renato Diniz Silveira ${ }^{1}$.

\section{RESUMO}

Objetivo: Demonstrar como as Diretrizes Curriculares Nacionais (DCNs) de 2014 foram aplicadas no processo de construção do Estágio Curricular Obrigatório em Saúde Mental, do curso de Medicina, em uma Instituição de Ensino Superior. Revisão bibliográfica: O Brasil ocupa, atualmente, o $5^{\circ}$ lugar no ranking de países com maiores taxas de depressão do mundo e uma incidência de $23,93 \%$ de algum tipo de transtorno de ansiedade entre os brasileiros. Frente a este cenário, novas estratégias têm sido buscadas para enfrentamento da alta quantidade de adoecimento psíquico na população. Em virtude dessas novas demandas, várias mudanças no processo de formação do profissional médico foram requeridas. Assim, os pilares da educação médica no Brasil, que até então eram normatizados e estruturados pelas DCNs de 2001, foram substituídos pelas DCNs de 2014. Considerações finais: O sofrimento psíquico, que anteriormente era subestimado, tornou-se prioridade no cuidado médico. Por essa razão, é essencial a garantia de uma formação sensível à saúde mental para os novos médicos. Este artigo almeja auxiliar outras instituições a construírem suas próprias matrizes pedagógicas em Saúde Mental com base nas DCNs de 2014.

Palavras-chave: Saúde mental, Educação médica, Internato médico, Reforma psiquiátrica.

\begin{abstract}
Objective: To demonstrate how the 2014 National Curricular Guidelines (NCGs) was applied on the Mental Health obligatory curricular internship, on a Medical University. Bibliographic review: Brazil occupies, actually, the 5th on the biggest depression rate ranking around the world and a $23.93 \%$ incidence of any kind of anxiety disorders among Brazilian citizens. Therefore, new strategies have been searched to cope with the high amount of psychic illness on that population. Thus, a lot of changes on the medical graduation process, have been necessary. So, the Brazilian medical education bases, that until then was regulated and structured by the 2001 NCGs, was substituted by the 2014 NCGs. Final Considerations: Psychic illness, which was previously underestimated, became a priority on the medical assistance. Therefore, it is essential that the medical graduation process approaches the mental health care qualifying the new physicians. The article aims to help other medical universities to build their own Mental Health programs based on the 2014 NCGs.
\end{abstract}

Keywords: Mental health, Medical education, Medical internship, Psychiatric reform.

1 Pontifícia Universidade Católica de Minas Gerais (PUC Minas), Betim - MG.

*E-mail: fernanda12penido@gmail.com

SUBMETIDO EM: 10/2021

PUBLICADO EM: 10/2021 


\section{RESUMEN}

Objetivo: Demostrar cómo se aplicaron las Directrices Curriculares Nacionales (DCNs) de 2014 en el proceso de construcción de las Prácticas Curriculares Obligatorias en Salud Mental de la carrera de Medicina en una Institución de Educación Superior. Revisión bibliográfica: Brasil ocupa actualmente el 5oㅡㄴ lugar entre países con mayores tasas de depresión en el mundo y $23,93 \%$ de incidencia de algún tipo de trastorno de ansiedad entre los brasileños. Ante este panorama, se han buscado nuevas estrategias para hacer frente al elevado número de enfermedades psíquicas en la población. Debido a estas nuevas exigencias, fue necesario introducir varios cambios en el proceso de formación de los profesionales médicos. Así, los pilares de la educación médica en Brasil, que hasta entonces estaban regulados y estructurados por las DCN de 2001, fueron sustituidos por las DCN de 2014. Consideraciones finales: sufrimiento psíquico, que antes se subestimaba, se ha convertido en una prioridad en la atención médica. Por este motivo, es esencial garantizar una formación sensible a la salud mental para nuevos médicos. Este artículo pretende ayudar otras instituciones a construir sus propias matrices pedagógicas en Salud Mental basadas en las DCN de 2014.

Palabras clave: Salud mental, Educación médica, Pasantía médica, Reforma psiquiátrica.

\section{INTRODUÇÃO}

Em todo o mundo avalia-se que haja 322 milhões de pessoas portadoras de depressão, cerca de $18 \%$ a mais do que nos últimos dez anos, além de 264 milhões com algum transtorno de ansiedade, um acréscimo de $14,9 \%$ em relação à última década. Se forem considerados os demais transtornos mentais conhecidos, estima-se que esses ocasionam uma perda global econômica em torno de US $\$ 1$ trilhão por ano em todo o planeta. No Brasil, o panorama da saúde mental também mostra-se significativo. O país ocupa, atualmente, o $5^{\circ}$ lugar no ranking de países com maiores taxas de depressão do mundo, com uma prevalência de 5,8\% em sua população. Além disso, contabiliza-se entre os brasileiros, uma incidência em torno de $23,93 \%$ de algum tipo de transtorno de ansiedade (WORLD HEALTH ORGANIZATION, 2017).

Soma-se a esses dados, o contexto atual no qual vivemos, o enfrentamento à pandemia de COVID-19. Embora não seja um contexto inédito na história da humanidade e apesar deste mesmo fato, sabemos que uma situação de pandemia compromete toda a população sendo, uma de suas contribuições negativas, suas consequências na saúde mental. Cenários como este aumentam os níveis de transtornos depressivos, níveis de stress e ansiedade, além de hábitos nocivos como tabagismo, etilismo e uso de drogas. Frente a esta conjuntura, torna-se importante e necessária a formação de profissionais que estejam aptos a contribuir de forma positiva na abordagem integral de cada indivíduo, o que inclui o bom manejo da saúde mental (FARO A, et al., 2020).

Em vista de tudo isso, no Brasil, o Sistema Único de Saúde (SUS), atualmente tem empregado como componente estratégico para enfrentamento da alta quantidade de adoecimento psíquico na população, a Reforma Psiquiátrica como norteadora do cuidado à pessoa com sofrimento mental. Esta reforma consiste em um modo de assistência que, desde 1980, propõe um modelo de atenção à saúde mental, baseado na reinserção social, na desinstitucionalização, na defesa da dignidade humana, da autonomia e do convívio social como parte central da assistência, em detrimento ao tratamento em hospitais psiquiátricos, que é baseado principalmente na internação involuntária (FARINHA MG e BRAGA TBM, 2018).

Em virtude das novas demandas técnicas e sociais da sociedade, especialmente da atenção à saúde, várias mudanças no processo de formação do profissional médico foram requeridas. Neste contexto, os pilares da educação médica no Brasil, que até então eram normatizados e estruturados pelas Diretrizes Curriculares Nacionais (DCN) de 2001, foram substituídos pelas DNC de 2014. Assim, de acordo com as mais recente $\mathrm{DCN}$, o graduado em medicina deverá ter formação geral, humanista, crítica, reflexiva e ética, sendo capaz de atuar em diversos níveis da atenção à saúde, com responsabilidade social e compromisso com a defesa da cidadania e da dignidade humana (MINISTÉRIO DA EDUCAÇÃO (MEC), 2001; MEC, 2014; ROCHA VXM, et al., 2017). 
Para concretização dessas habilidades demandadas pelas novas DNC, houve a inclusão do internato em Saúde Mental como atividade obrigatória dos cursos de medicina. Essa mudança vai ao encontro do contexto atual de emprego da Reforma Psiquiátrica, da significativa prevalência de agravos relacionados à saúde mental na população e da decorrente necessidade de sua assistência (ROCHA VXM, et al., 2017).

A introdução da Saúde Mental no curso de Medicina ocorreu baseada no pressuposto de que o profissional médico capacitado a assistir as pessoas com adoecimento mental deve compreender 0 sofrimento em suas dimensões culturais, sociais, econômicas, biológicas e psicológicas. Assim sendo, 0 generalista dentro do contexto da Atenção Primária (AP), estaria apto a resolver as principais e mais frequentes queixas e situações da saúde mental, articulando com as diversas ferramentas do SUS, suas particularidades, potencialidades e fluxos (ROCHA VXM, et al., 2017).

Apesar das mudanças curriculares estarem em voga desde 2014, observa-se que muitas escolas médicas ainda apresentam dificuldades na implantação e na adequação das determinações das DCN. As estimativas de 2019 apontam que a inclusão da Saúde Mental no internato era ausente em até metade dos centros médicos de ensino (CÂNDIDO PTS e BATISTA NA, 2019).

Em contraposição a este cenário, o curso de Medicina de uma universidade privada de Minas Gerais, iniciado em 2012, incorporou o internato de Saúde Mental em sua grade curricular, antes mesmo das DCN de 2014, devido a deflagrante demanda e necessidade de assistência adequada ao paciente com sofrimento mental. Além das exigências curriculares tradicionais, o curso em questão adotou para uma formação mais humanizada de seus discentes, o Programa de Mentoring, que estimula a essência do cuidado. Assim, a grade curricular do curso tem passagem pelas ciências humanas, psicologia, relação médico-paciente e psiquiatria, dispondo de uma importante formação em Saúde Mental, em conformidade com a DCN e ao mesmo tempo, respondendo aos anseios do movimento da reforma psiquiátrica brasileira (SILVEIRA RD, 2016; SILVEIRA RD, et al.,2021).

Portanto, sabendo-se que a Universidade deve ser uma instituição que incorpora o ensino em saúde mental ao curso de medicina, o presente artigo teve como objetivo trazer uma breve revisão narrativa da matriz de ensino curricular do curso de Medicina e do plano de ensino da disciplina do Internato de Saúde Mental da instituição em questão, para elucidar a importância e o impacto de uma formação acadêmica dentro das necessidades assistenciais no campo da saúde mental para o futuro médico generalista.

\section{REVISÃO BIBLIOGRÁFICA}

\section{Saúde mental no ensino médico e contexto}

O ensino médico no Brasil é regido, atualmente, pelas DCNs determinadas pela Resolução №3 do Conselho Nacional de Educação, de 20 de junho de 2014 que substituiu a Resolução $N^{\circ}$ 4, de 7 de novembro de 2001. Diferentemente da anterior, a resolução atual estabeleceu, dentre algumas mudanças, uma formação que além dos aspectos biológicos, psicológicos, éticos, socioeconômicos, ambientais e culturais, fosse, ainda, baseada na responsabilidade social, no respeito aos direitos humanos, à dignidade e à saúde integral do indivíduo. Ademais, a resolução visa corrigir o descompasso da formação médica baseada no modelo de atenção à saúde criado no século XX para atender às condições agudas e infectoparasitárias, atualizando-a para assistir às novas demandas em saúde contemporâneas, as quais são centradas em condições, essencialmente, crônicas (MEC, 2001; MEC, 2014; MINISTÉRIO DA SAÚDE, 2015; CÂNDIDO PTS e BATISTA NA, 2019).

Para além das transições epidemiológicas e demográficas, a Resolução № 3 das DCNs passa a incorporar, no processo de construção do currículo médico, competências que permitam ao futuro profissional concretizar os princípios do SUS e a estabelecer, como requisito no processo de aprendizado do cuidado com o usuário, a assistência centrada na pessoa, na família e na comunidade, com o desenvolvimento de uma relação horizontal (MEC, 2014).

Ao encontro das mudanças estabelecidas pela nova DCN, a Resolução n³/2014 estabelece a implantação do Internato em Saúde Mental, como uma área obrigatória dentre as atividades do regime de 
internato médico da Graduação em Medicina que deverá totalizar no mínimo 35\% da carga horária total do curso. Essa medida, em grande parte, foi resultado da Lei no 12.871, de 2013, que instituiu o Programa Mais Médicos com foco na atenção básica, de modo com que houvesse a necessidade de adaptação das DCNs do ensino médico, especialmente no que diz respeito à disjunção da área da Psiquiatria da área de Clínica Médica. Assim, adotando o conceito de Saúde Mental como uma estratégia que possibilita ao médico reconhecer e agir sobre o sofrimento mental humano na AP, sem considerá-lo meramente como um diagnóstico psiquiátrico destinado apenas à atenção secundária (MEC, 2014; CAVALCANTI MT, et al., 2019; SECRETARIA DE ESTADO DE SAÚDE, 2006; PAULIN LF, 2020).

Deve-se reforçar que o internato em discussão tem seus fundamentos no conceito de Saúde Mental, portanto, não se limita ao ensino puramente da psiquiatria. Assim, cabe esclarecer que os termos - saúde mental e psiquiatria - não são sinônimos ou substitutos, mas pertencem a um campo comum de conhecimento e de práticas, sendo o primeiro voltado à prevenção e à promoção de saúde e o último como o núcleo de saber que reconhece e trata os transtornos mentais (FIGUEIREDO FP, et al., 2019).

Com isso, o médico deixa o papel de centralizador no processo saúde-doença e no tratamento do paciente, em prol da necessidade de intersetorialidade de cuidado da doença mental, haja visto a limitação deste profissional como responsável único pelo paciente. Sendo assim, encarregado pelo direcionamento de terapêuticas, bem como o estabelecimento de hipóteses diagnósticas e orientações psicofarmacológicas, em assistência compartilhada com os demais profissionais da equipe, assim como o usuário e sua família (FIGUEIREDO FP, et al., 2019).

As mudanças curriculares advindas da DCN de 2014 ocorreram, ainda, em convergência aos princípios da Estratégia de Saúde da Família (ESF) implantada em 2014, sendo, atualmente, o principal instrumento de assistência populacional em saúde do Brasil. Ademais, a nova resolução tornou-se, também, alinhada com as leis e com as regulamentações resultantes do movimento de Luta Antimanicomial, bem como com os princípios e com as diretrizes da Reforma da Assistência Psiquiátrica Brasileira, as quais contribuíram para a mudança no modelo do ensino médico na saúde mental. Este modelo passa a ser pautado em uma forma de assistência descentralizada, desospitalizada, pautada na instalação de uma rede de serviços e estratégias familiares e comunitárias, baseada na escuta e no acolhimento (MEC, 2014; FERREIRA MJM, et al., 2019; PARREIRA BDM, et al. 2017; PEREIRA AA e ANDRADE DCL, 2018).

Dentre as competências que se espera do médico generalista, está a capacidade de reconhecer as vulnerabilidades que abrangem determinados grupos sociais e, a partir disso, desenvolver e manter uma relação médico-paciente pautada no entendimento da complexidade que envolve o tratamento e 0 acompanhamento dos chamados Transtornos Mentais Comuns (TMC). Os TMC são manifestações de sintomas mistos de ansiedade, depressão, insônia e sintomas somáticos, com prevalência de até $64,4 \%$ no contexto da AP no Brasil. Diante disso, a implantação do Internato em Saúde Mental na grade curricular se apresenta como uma estratégia para a formação médica consoante com as demandas atuais da sociedade, visto que os TMC são altamente prevalentes e exigem conhecimentos e habilidades específicas (MEC, 2014; BALLESTER 2005; GONÇALVES DA, et al., 2014; PEREIRA AA e ANDRADE DCL, 2018).

No âmbito do ensino da saúde mental, a AP recebe, também, de forma progressiva, os pacientes que são reinseridos na sociedade progressivamente após a Reforma Psiquiátrica Brasileira, sendo responsável pelo seu acompanhamento e assistência em saúde. A Resolução no3 da DCN é assertiva em suas determinações legais ao considerar que os campos de prática dos internatos médicos sejam garantidos dentro da AP por meio do Contrato Organizativo de Ação Pública Ensino-Pesquisa (COAPES), uma vez que este é o nível de complexidade mais resolutivo da rede em atenção em saúde. Além disso, a AP é responsável pela maior parte da assistência à saúde populacional, incluindo as demandas em saúde mental, sendo assim o campo mais adequado para o treinamento do interno (MEC, 2014; CÂNDIDO PTS e BATISTA NA, 2019).

Ainda que as novas DCN determinem $35 \%$ da carga horária do curso seja voltada para o internato e que a carga teórica não deve ultrapassar $20 \%$ do tempo total em cada disciplina, dados da literatura apontam 
que os médicos da AP não se sentem preparados para atender com qualidade e resolutividade os casos de transtornos mentais prevalentes na comunidade. Paralelamente, nos últimos 20 anos, o número de escolas médicas triplicou no Brasil e, em 2020, o país contava com 351 cursos de Medicina autorizados pelo Ministério da Educação, o que aponta a necessidade de monitoramento das estratégias educacionais utilizadas pelas escolas médicas brasileiras (CÂNDIDO PTS e BATISTA NA, 2019; GONÇALVES DA, et al., 2014; PEREIRA DVR, et al. 2020).

\section{A matriz de saúde mental}

Conforme previamente exposto, a antiga DNC de 2001, era vaga no que diz respeito às atribuições da instituição de ensino superior para garantir a formação de seus graduandos para atuação na Saúde Mental. O texto vinculava-se apenas ao campo da psiquiatria, abordando apenas citações esparsas de que o estudante de Medicina deveria estar apto para compreender o paciente em sua totalidade. Com as novas DCNs, vigentes desde 2014, além de trazer em todo o seu escopo um destaque maior à Saúde Mental, estabelece a obrigatoriedade do estágio curricular em Saúde Mental e reforça a importância do graduando em medicina receber ensino adequado nessa área (MEC, 2001; MEC, 2014; PAULIN LF, 2020).

A mais recente DNC determinou que os cursos de Medicina iniciados antes de 2014 teriam até 31 de dezembro de 2018 para se adequarem progressivamente à nova proposta. Isso porque mudanças consideráveis são notadas entre as duas diretrizes, sobretudo em relação às determinações quanto à macroárea da Saúde Mental. Para isso o graduando deve ser capaz de compreender os determinantes sociais, culturais, comportamentais, psicológicos, ecológicos, éticos e legais, nos âmbitos individuais e coletivos da saúde. Além disso, ele deve dominar a propedêutica médica: capacidade de realizar história clínica, exame físico, conhecimento fisiopatológico dos sinais e sintomas, capacidade reflexiva e compreensão ética e humanística da relação médico-pessoa sob cuidado (MEC, 2014; PAULIN LF, 2020).

\section{O plano de ensino da instituição}

No ano de 2016, foi redigida e implementada a Matriz na área da Saúde Mental, referente ao currículo do curso de Medicina da universidade aqui tratada. O objetivo fundamental dessa matriz é garantir a formação em saúde mental dos estudantes, visando a atuação dos mesmos como médicos generalistas capazes de ir além da exploração de sinais, sintomas e tratamentos (FIGUEIREDO FP, et al., 2019; SILVEIRA RD, 2016; SILVEIRA RD, et al., 2021).

O Programa de Mentoring, no qual um mentor acompanha uma turma ao longo de todo curso, foi instituído como atividade obrigatória para potencializar a formação dos alunos. Com essa prática, é estabelecida uma cultura de cuidado, que considera os aspectos socioculturais, psicofisiológicos e as experiências individuais. O mentor, por meio de encontros em grupos e individuais, auxilia na resolução de dificuldades, reflete e promove acolhimento aos discentes ao longo dos 6 anos de curso. Assim, as noções de cuidado são vivenciadas enquanto alunos para serem colocadas em prática futuramente como médicos. Para a promoção de mudanças no trabalho médico e relação médico-paciente devem haver mudanças primeiramente na dinâmica do ensino-aprendizagem médica (SILVEIRA RD, et al.,2021).

Nesse sentido, tem-se, como objetivos específicos, que os alunos graduados em medicina por essa universidade sejam preparados para realizar a anamnese e o exame mental psiquiátricos, com a elaboração de hipóteses diagnósticas e diagnósticos diferenciais em psiquiatria e com destaque para o tratamento psicofarmacológico e outras estratégias de tratamento adotadas no campo da saúde mental. Além disso, propõe-se o treinamento de habilidades em urgências e emergências em psiquiatria e que os futuros médicos estejam aptos para solucionar as principais e mais frequentes queixas no campo da saúde mental no nível da AP (CAMPOS GWS, 2017; FIGUEIREDO FP, et al., 2019; SILVEIRA RD, 2016).

É relevante destacar, também, as propostas para que os estudantes adquiram competências para: identificar os principais problemas advindos da área da dependência química, fazendo projetos terapêuticos em equipe e considerando todo o contexto social e familiar em que o paciente está inserido; ir além da anamnese convencional ao entrevistar um indivíduo, contextualizando a queixa principal do mesmo em sua 
história de vida; formar e consolidar a competência de trabalhar em equipe, levando em consideração o saber de todos os trabalhadores envolvidos na saúde mental, o diálogo com os familiares dos pacientes, articulando os desafios e potencialidades que estes têm no tratamento dos mesmos; saber articular o caso clínico com os aspectos intersetoriais envolvidos, o que vai ao encontro do que é preconizado pelos princípios da reforma psiquiátrica (FIGUEIREDO FP, et al., 2019; MINISTÉRIO DA SAÚDE, 2005)

Frente a isso, desde o primeiro período do curso de Medicina, foram inseridas disciplinas que pudessem proporcionar o saber necessário para que os objetivos descritos acima fossem alcançados. No Ciclo Básico (1 ao $4^{\circ}$ período) as disciplinas "Ciências Sociais Aplicadas à Saúde", "Filosofia: antropologia e ética" e "Filosofia: razão e modernidade" são importantes para despertar nos estudantes, reflexões acerca das sociedades e das culturas. Já as disciplinas de imersão no ambiente prático do SUS, nas quais se encontram as Unidades Básicas de Saúde (UBSs), os Centros de Atenção Psicossocial (CAPS), assim como as "Práticas na Comunidade", são essenciais para que os futuros médicos conheçam a organização, a estrutura e o funcionamento do Sistema Único de Saúde (SUS) (SILVEIRA RD, 2016).

A partir do Ciclo Clínico (5ำ ao $8^{\circ}$ período) o aluno passa a ter um contato direto com temas muito frequentes na área da Saúde Mental. Isso se inicia com a disciplina "Introdução à Saúde Mental", na qual o graduando é introduzido à temática da psicanálise. Já na "Semiologia Geral", o discente desenvolve suas habilidades relacionadas a anamnese médica, além de desfrutar da semiologia específica da psiquiatria, quando aprende a fazer o exame psiquiátrico básico e a avaliação psiquiátrica em pacientes da AP e dos Centros de Atenção Psicossocial. Ademais, no 8 período do curso, é ofertada a matéria "Saúde Mental", por meio da qual os alunos são capacitados para realizar hipóteses diagnósticas em psiquiatria, articulando as mesmas com diversos aspectos do campo da saúde mental (SILVEIRA RD, 2016).

Por fim, no período do internato, os alunos estão aptos para colocar em prática, sob supervisão, os conhecimentos teórico-práticos adquiridos nos ciclos anteriores, fazendo uso destes no cotidiano da prática médica. Os alunos são divididos em grupos de 5 a 6 pessoas para que os discentes possam realizar rodízio entre os cenários de prática, nos quais o interno permanece por no mínimo três semanas, em imersão de 28 horas semanais, divididos por turnos de quatro horas. Cada grupo passa ao todo por três campos, totalizando um mínimo de 9 semanas (SILVEIRA RD, 2016).

As atividades realizadas no internato envolvem: acompanhamento de casos clínicos com apresentação e devolutiva para a equipe; visitas domiciliares e discussões intersetoriais; anotações em prontuários e registros das ações realizadas; participação na escala do serviço e evolução dos pacientes; articulação com o matriciamento em saúde mental; visitas técnicas ao CAPS Infanto-juvenil; atendimento supervisionado em UBSs; participação em oficinas terapêuticas e ações de reabilitação psicossocial; construção de projeto terapêutico individual; atendimento supervisionado ao dependente químico e sua família; trabalho ativo nas altas hospitalares e articulação do indivíduo com serviço substitutivo. Dessa forma, busca-se compreender todo o contexto biopsicossocial do indivíduo e articulá-lo com a Atenção Primária, assim aumentando suas redes de apoio (CAMPOS GWS, 2017; SILVEIRA RD, 2016).

Em cada campo de estágio os internos são supervisionados por 2 professores, sendo um psiquiatra e um psicólogo. Assim sendo, além das atividades práticas realizadas em cada campo, semanalmente são realizados dois grupos de discussão com temas os quais contemplam tanto a psiquiatria quanto a psicologia. Os temas discutidos incluem: esquizofrenia; transtorno de ansiedade; transtornos de humor; diagnóstico estrutural em psicanálise; atenção aos aspectos da Saúde Mental da criança e do adolescente; estudo de apoio matricial em Saúde Mental; acompanhamento terapêutico e oficinas terapêuticas; antidepressivos; antipsicóticos; estabilizadores de humor; ansiolíticos e hipnóticos; abordagem e manejo da crise de abstinência alcoólica e psicofarmacologia em situações especiais (SILVEIRA RD, 2016).

Por fim, fora do espaço dos campos de estágio, os internos recebem aulas teóricas ministradas pelos professores da disciplina que versam sobre os temas: dependência química e toxicomania; redução de danos; Saúde Mental na atenção básia; princípios da psicofarmacologia; tratamento da dependência química; e oficinas terapêuticas na Saúde Mental (SILVEIRA RD, 2016). 


\section{CONSIDERAÇÕES FINAIS}

O sofrimento psíquico, anteriormente subestimado, tornou-se prioridade no cuidado médico. Por essa razão, é essencial a garantia de uma formação sensível à saúde mental na formação médica. Para isso, devem ser traçadas estratégias no currículo acadêmico do curso de Medicina que eliminem estigmas e preconceitos relacionados ao paciente de saúde mental. Dessa forma, o acadêmico de medicina deve possuir em sua formação princípios humanos e de responsabilidade social para que se esforcem na garantia da cidadania de seus pacientes, conforme já determinado na DCN. Assim, com este trabalho, espera-se que as escolas de medicina do país tenham subsídios na referência científica para busca de experiências na implementação e estruturação das DCN de 2014 no que se refere à Saúde Mental.

\section{REFERÊNCIAS}

1. BALLESTER DA, et al. The general practitioner and mental health problems: challenges and strategies for medical education. Sao Paulo Medical Journal, 2005; 72-76.

2. CAMPOS GWS. Papel da rede de atenção básica em saúde mental na formação médica - diretrizes. Cad ABEM, 2007; 3: 6-10.

3. CÂNDIDO PTS, BATISTA NA. O Internato Médico após as Diretrizes Curriculares Nacionais de 2014: um Estudo em Escolas Médicas do Estado do Rio de Janeiro. Revista. Brasileira de Educação Médica, 2019; 43 (3): 36-45.

4. CAVALCANTI MT, et al. Internato de Saúde Mental para alunos de Medicina: qual o melhor cenário de formação? Interface, 2020; 24: 1 -13.

5. FARINHA MG, BRAGA TBM. Sistema único de saúde e a reforma psiquiátrica: desafios e perspectivas. Revista da Abordagem Gestáltica: Phenomenological Studies, 2018; 24 (3): 366-378.

6. FARO A, et al. COVID-19 e saúde mental: a emergência do cuidado. Rev. Estud. Psicol., 2020, 37.

7. FERREIRA MJM, et al. Novas Diretrizes Curriculares Nacionais para os cursos de Medicina: oportunidades para ressignificar a formação. Rev. Interface, 2019; 23 (Supl. 1): 1-15.

8. FIGUEIREDO FP, et al. A trajetória da implantação de um internato de Saúde Mental em uma instituição de ensino superior. Interface, 2019; 23 (supl.1): 1-12.

9. GONÇALVES DA, et al. Brazilian multicentre study of common mental disorders in primary care: rates and related social and demographic factors. Cad. Saúde pública, 2014; 30(03): 623-632.

10. MINISTÉRIO DA EDUCAÇÃO (MEC). Parecer homologado 116/2014 D.O.U. Brasil, 2014: 17. Disponível em: http://portal.mec.gov.br/index.php?option=com_docman\&view=download\&alias=15514-pces 116$14 \&$ category slug=abril-2014-pdf\&Itemid=30192. Acessado em 30 de set. de 2021.

11. MINISTERIO DA EDUCAÇÃO (MEC). Resolução no3, Brasil, $2014 . \quad$ Disponível em: http://portal.mec.gov.br/index.php?option=com_docman\&view=download\&alias=15874-rces00314\&category_slug=junho-2014-pdf\&ltemid=30192. Acessado em 30 de set. de 2021.

12. MINISTERIO DA EDUCAÇÃO (MEC). Resolução no ${ }^{2}$ 4, Brasil, 2001. Disponível em: http://portal.mec.gov.br/cne/arquivos/pdf/CES04.pdf. Acessado em 30 de set. de 2021.
MINISTÉRIO DA SAÚDE. Análise de Situação de Saúde (ASIS). $2015 . \quad$ Disponível em:

13. MINISTÉRIO DA SAÚDE. Análise de Situação de Saúde (ASIS). 2015 . Disponível em:
https://bvsms.saude.gov.br/bvs/publicacoes/asis_analise_situacao_saude_volume_1.pdf. Acessado em 30 de set. de 2021.

14. MINISTÉRIO DA SAÚDE. Reforma psiquiátrica e política de saúde mental no Brasil. 2005. Disponível em: https://bvsms.saude.gov.br/bvs/publicacoes/Relatorio15_anos_Caracas.pdf. Acessado em 30 de set. de 2021.

15. PARREIRA BDM, et al. Transtorno mental comum e fatores associados: estudo com mulheres de uma área rural. Rev Esc Enferm USP, 2017; 51: 1-8.

16. PAULIN LF, et al. Construindo o Internato de Saúde Mental: a Experiência da Universidade São Francisco. Rev. bras. educ. med, 2020; 44 (1).

17. PEREIRA AA, ANDRADE DCL. Estratégia Educacional em Saúde Mental para Médicos da Atenção Básica. Rev. bras. educ. med, 2018; $42(1): 1-12$.

18. PEREIRA DVR, et al. Cartografia das escolas médicas: a distribuição de cursos e vagas nos municípios brasileiros em 2020. Rev. bras. educ. med, 2021; 45 (1): 1-10.

19. ROCHA VXM, et al. Reformas na educação médica no Brasil: estudo comparativo entre as diretrizes curriculares nacionais dos cursos de graduação em medicina de 2001 e 2014. Dissertação (Mestrado em Saúde Coletiva) Políticas de Saúde. Universidade Católica de Santos, Santos, 2018: 178.

20. SECRETARIA DE ESTADO DE SAÚDE. Linha Guia da Saúde Mental. Belo Horizonte, Minas Gerais, 2006 Disponível em: https://psiquiatriabh.com.br/wp/wp-content/uploads/2015/01/Linha-guia-de-saude-mental.pdf. Acessado em: 18 de outubro de 2021.

21. SILVEIRA RD. Matriz do curso de medicina da PUC Minas. Área: Saúde Mental. 2016.

22. SILVEIRA RD, et al. O Programa de Mentoring da PUC Minas: relato de experiência. Revista Brasileira de Educação Médica, 2021; 45(1): e110.

23. WORLD HEALTH ORGANIZATION (WHO). Depression and Other Common Mental Disorders: Global Health Estimates. 2017. Disponível em: https://www.who.int/publications/i/item/depression-global-health-estimates. Acessado em 30 de set. de 2021.

24. WORLD HEALTH ORGANIZATION (WHO). Mental Health: strengthening our response. 2018. Disponível em: https://www.who.int/news-room/fact-sheets/detail/mental-health-strengthening-our-response. Acessado em 30 de set. de 2021. 